\title{
Revealing Partons in Hadrons: From the ISR to the SPS Collider
}

\author{
Pierre Darriulat ${ }^{1}$ and Luigi Di Lella ${ }^{2}$ \\ ${ }^{1}$ VATLY, INST, 179 Hoang Quoc Viêt, Cau Giay, Ha Noi, Viêt Nam \\ ${ }^{2}$ Università di Pisa, Physics Department, Largo Bruno Pontecorvo 3, \\ 56127 Pisa, Italy \\ pierre.darriulat@gmail.com
}

\begin{abstract}
Our understanding of the structure of hadrons has developed during the seventies and early eighties from a few vague ideas to a precise theory, Quantum Chromodynamics, that describes hadrons as made of elementary partons (quarks and gluons). Deep inelastic scattering of electrons and neutrinos on nucleons and electron-positron collisions have played a major role in this development. Less well known is the role played by hadron collisions in revealing the parton structure, studying the dynamic of interactions between partons and offering an exclusive laboratory for the direct study of gluon interactions. The present article recalls the decisive contributions made by the CERN Intersecting Storage Rings and, later, the proton-antiproton SPS Collider to this chapter of physics.
\end{abstract}

\section{Preamble}

In the mid-sixties, when the ISR were being born, the idea that hadrons could be composite particles was still far from being generally accepted. Summer school lectures were giving as much weight to bootstrap ideas ${ }^{1}$ as to the newly born quark model. ${ }^{2}$ We remember a seminar by C. N. Yang ${ }^{3}$ at CERN, just before the ISR first collisions, introducing the concept of limiting fragmentation, which we were religiously listening to in the hope that it could give us an idea of what to expect from our imminent exploration of the high energy territory. In spite of the spectacular success of Gell-Mann's eightfold way, the quark model had to face two very strong counter-arguments: the failure of many quark search experiments to find any hint for fractional charges and the apparent incompatibility of the quark model with Fermi-Dirac statistics. Indeed, we did not know about colour, nor about the peculiar behavior of the strong force to get weaker at short distances. The light would come from SLAC at the very end of the decade, with deep inelastic electron scattering soon followed by SPEAR and its harvest of revolutionary results.

If hadrons are composite, it should be possible to understand hadron collisions in terms of interactions and rearrangements of the constituents, the so-called partons, and, in particular, to eject one of them, as in nuclear physics with $(p, 2 p)$ or $(p, p n)$ reactions. It is indeed possible, but it took a decade to reach this goal. Hadrons are very different from nuclei, which can be qualitatively described classically in 
this context. For two main reasons: one is that hadron masses are much larger than parton masses, making the picture fully relativistic; the other is the increase of the strength of the strong force with distance, making it impossible to eject an isolated parton: as it is pulled apart from its parent hadron, the field of the strong force in between takes such high values that quark-antiquark pairs are produced in the form of mesons that accompany the ejected parton. To identify such a collection of hadrons as the filiation of the parent parton among the host of other hadrons produced in the collision is only possible when they fly close enough together to form what is called a jet. In what follows, we try to recall how this was achieved at CERN, from the ISR to the proton-antiproton SPS collider, between the early seventies and the mid-eighties. Most of it is borrowed from two earlier papers of ours. ${ }^{4,5}$

\section{The ISR as a Gluon Collider}

\subsection{Introduction}

It so happens that the lifetime of the ISR, roughly speaking the seventies, coincides with a giant leap in our understanding of particle physics. However, it is honest to say that, to first order, there is no causal relation between the two. Yet, those of us who have worked at the ISR remember these times with the conviction that we were not merely spectators of the ongoing progress, but also - admittedly modest actors. The ISR contribution, it seems to us, is too often unjustly forgotten in the accounts that are commonly given of the progress of particle physics during this period. We shall try to present arguments of relevance to this issue in what we hope to be as neutral and unbiased way as possible. We restrict the scope of the presentation to large transverse momentum processes, or equivalently to the probing of the proton structure at short distances. This, however, is not much of a limitation, as the ISR did not significantly contribute to the progress achieved in the weak sector.

Each individual has his own vision of the past and history can merely be an attempt at collecting all such visions into as coherent as possible a story. In physics, this is particularly true when discoveries and new ideas occur at a rapid pace, as was the case in the seventies. Each of us remembers a seminar, a discussion at coffee, the reading of a particular article, or another event of this kind as a milestone in his own understanding of the new ides. Reading accounts by Steve Weinberg, ${ }^{6}$ David Gross, ${ }^{7}$ Gerard 't Hooft ${ }^{8}$ or Jerry Friedman' ${ }^{9}$ of how they remember this period is particularly instructive in this respect. The same kind of disparity that exists between the visions of different individuals also occurs between the visions of different science communities. In particular, during the seventies, the $e^{+} e^{-}$, neutrino, fixed target and ISR communities had quite different perceptions of the progress that was being achieved. It is therefore useful to recall briefly the main events in this period. 


\subsection{The main milestones}

When Vicky Weisskopf, in December 1965, in his last Council session as CERN Director-General obtained approval for the construction of the ISR, there was no specific physics issue at stake, which the machine was supposed to address; its only justification was to explore the terra incognita of higher centre-of-mass energy collisions (to our knowledge, since then, all new machines have been proposed and approved with a specific physics question in mind, which they were supposed to answer). The strong interaction was perceived as a complete mystery. The eightfold way, today understood as the approximate $S U(3)$ flavour symmetry associated with interchanges of $u, d$ and $s$ quarks, was not believed to have significant consequences in the dynamics of the strong interaction. The fact that no free quark had been found in spite of intensive searches, and that states such as $\Delta^{++}$, with spin-parity $3 / 2^{+}$, could not be made of three identical spin $1 / 2 u$ quarks without violating Fermi statistics, were discouraging such interpretations.

The first hint to the contrary came in 1968-1969 at SLAC ${ }^{9}$ with the discovery of an important continuum in the deep inelastic region of electron proton scattering. The 2-mile linear accelerator had started operation the preceding year and the experimental program, using large spectrometers, extended over several years. From the very beginning, experimenters and theorists were in close contact, feeding each other with new data and new ideas, starting with Bjorken's ideas on scaling ${ }^{10}$ and Feynman's ideas on partons, ${ }^{11}$ both early advocates of a proton structure consisting of point-like constituents. However, one had to wait until 1972 for the case for a quark model to become strong: by then, scaling had been established; the measurement of a small $R$ value (the ratio of the absorption cross sections of transverse and longitudinal virtual photons) had eliminated competitors such as the then popular Vector Dominance Model; deuterium data had been collected allowing for a comparison between the proton and neutron structure functions; a number of sum rules had been tested; evidence for the quarks to carry but a part of the proton longitudinal momentum had been obtained; the first neutrino deep inelastic data from Gargamelle had become available. ${ }^{12}$ By the end of 1972 , the way was paved for Gross, Wilczek and Politzer ${ }^{13}$ to conceive the idea of asymptotic freedom and its corollary, infrared slavery, explaining why one could not see free quarks. By the end of 1973, the connection with non-Abelian gauge theories had been established and the "advantages of the colour-octet gluon picture", including the solution of the Fermi statistics puzzle, had been presented by Fritzsch, Gell-Mann and Leutwyler. ${ }^{14}$ QCD was born and, by 1974, was starting to be accepted by the whole community as the theory of the strong interaction. It took another three to four years for it to come of age.

By mid-1972, SPEAR, the Stanford electron-positron collider, had begun operation. In November 1974, it shook the physics community with what has since been referred to as a revolution: the discovery of the $\Psi$ going hand in hand with the simultaneous discovery of the $J$ at Brookhaven. It immediately exploited its 
ability to produce pure quark-antiquark final states to measure the number of colours. However, there were so many things happening in the newly available energy domain (opening of the naked charm channels, crowded charmonium spectroscopy, production of the $\tau$ lepton) that it took some time to disentangle their effects and to understand what was going on. By the end of the decade, scaling violations had been studied both in neutrino interactions and in electron-positron annihilations (DORIS had started operation in Hamburg two years after SPEAR). QCD had reached maturity and the only puzzling questions that remained unanswered, the absence of a CP violating phase and our inability to handle the theory at large distances, are still with us today.

\subsection{What about the ISR?}

The above account of the progress of particle physics in the seventies, while following the standard folklore, does not even mention the name of the ISR. Being asked whether he was aware of the results obtained at the ISR and whether they had an impact on the development of QCD, David Gross answered: ${ }^{15}$ "Every one was aware of the qualitative phenomena observed in hadronic physics at large $p_{T}$, which were totally consistent with simple scattering ideas and parton model ideas [...] The tests were not as clean as in deep inelastic scattering, the analysis was more difficult and deep inelastic scattering was much cleaner in the beginning of perturbative QCD [...] Parton ideas did not test QCD at all, they simply tested the idea that there were point-like constituents but not the dynamics." His answer illustrates well the way in which the ISR were generally perceived: a collider that was shooting Swiss watches against each other, as Feynman once jokingly described. Yet, some theorists followed closely what the ISR were producing; paradoxically, Feynman was one of them, Bjorken was another.

David Gross could have returned the question to us: "How aware were you, the ISR community, of the experimental progress at SLAC and of the new ideas in theory?" The first name that comes to mind in answer to this question is that of Maurice Jacob. Maurice had spent a sabbatical at Stanford where, together with Sam Berman, he had written a seminal paper on point-like constituents

and large transverse momentum production. ${ }^{16}$ Back at CERN, he organised a lively series of discussions between ISR experimenters and theorists that proved to be extremely successful in permeating our community with the progress in deep inelastic scattering and, later, in electron-positron collisions. At that time, our community was small enough to fit in the ISR auditorium. Maurice was gifted with an unusual talent to make theoretical ideas accessible to us. We all remember these seminars as a most profitable experience that brought coherence and unity in our community. For this reason, it makes sense to talk about a common ISR culture. In particular, by 1972, we were aware of the basic parton ideas and of the picture of large transverse momentum production factorised in three steps (Fig. 1): singling 


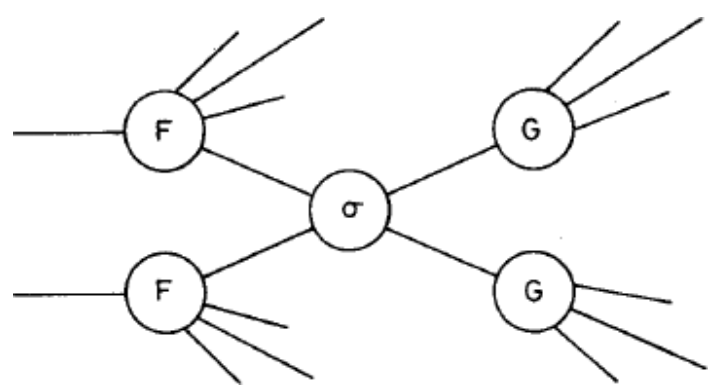

Fig. 1. Parton model picture of high $p_{T}$ hadron interactions. One parton of each of the incident hadrons (structure function $F$ ) experiences a binary collision $(\sigma)$ and the outcoming partons fragment into hadrons (fragmentation function $G$ ).

out a parton in each proton, making them interact (how, was not clear) in a binary collision and letting the final state partons fragment into hadrons. There were a few papers $^{11,16-21}$ in support of such a picture which most of us had read and which were our basic reference. Yet, in these early days, there was a typical delay of at least six months between SLAC and us for a new idea to be digested. There was even more delay, for most of us, to digest the subtle development of non-Abelian gauge theories: we only heard of them from our theorist friends.

Table 1 lists leading order diagrams involving quarks or gluons. A simple glance at it illustrates the originality of the ISR: gluons contribute to leading order. In electron-proton annihilations and deep inelastic scattering, gluons contribute to next to leading order only, in the form of radiative corrections associated with a bremsstrahlung gluon radiated from a quark line. This does not mean that such gluon contributions are unimportant: the scaling violations which they induce have been one of the most powerful tools in the development of our understanding of QCD. But, at the ISR, gluons not only contribute to leading order but indeed dominate the scene: in the low $x$ regime characteristic of the ISR, collisions involving gluons, either gluon-gluon or quark-gluon, account for most of the high $p_{T}$ crosssection. Gluon interactions being a privileged domain of the ISR, and gluons having been the last component of the theory to be understood and digested, it seems difficult to argue that the ISR have played but a minor role. The more so when one considers that the ISR had exclusive access to the three and four gluon vertices, a specific expression of QCD as a non Abelian gauge theory.

\subsection{Large transverse momentum: Inclusive production data}

In 1972-1973, three ISR teams ${ }^{22-24}$ announced the observation of an unexpectedly copious pion yield at large transverse momenta (Fig. 2), orders of magnitude above a (traditionally called naive) extrapolation of the exponential distribution observed at low $p_{T}$ values, $\sim \exp \left(-6 p_{T}\right)$. "Unexpectedly" is an understatement. The whole ISR experimental program had been designed under the assumption that all hadrons 
Table 1 Leading order processes involving quarks or gluons. The symbols $><$ and ][ stand for $s$ and $t$ channel exchange, respectively. The last column gives the coupling constants, the number of structure functions $(F)$ and the number of fragmentation functions $(G)$. Couplings are written $\alpha_{n}$ for $\alpha /\left(\sin \theta_{W} \cos \theta_{W}\right)^{2}$ and $\alpha_{c h}$ for $\alpha / \sin \theta_{W}^{2}$ with $\theta_{W}$ being the Weinberg angle. Processes involving gluons in the initial state are shaded.

\begin{tabular}{|c|c|c|c|}
\hline \multicolumn{4}{|c|}{ Electron-positron annihilations } \\
\hline 1 & & $\overline{e^{+} e^{-}>\gamma<q} \bar{q}$ & $\alpha^{2} G^{2}$ \\
\hline \multicolumn{4}{|c|}{ Deep inelastic electron scattering } \\
\hline 2 & & $e q] \gamma[e q$ & $\alpha^{2} F G$ \\
\hline \multicolumn{4}{|c|}{ Deep inelastic neutrino scattering } \\
\hline 3 & Neutral currents & $\nu q] Z[\nu q$ & $\alpha_{n}^{2} F G$ \\
\hline 4 & Charged currents & $\nu q] \mathrm{W}[l q$ & $\alpha_{c h}^{2} F G$ \\
\hline \multicolumn{4}{|c|}{ Proton-proton collisions (ISR) } \\
\hline 5 & Drell-Yan & $\overline{q \bar{q}>\gamma<l^{+} l^{-}}$ & $\alpha^{2} F^{2}$ \\
\hline 6 & Direct photons & $q \bar{q}] q[\gamma g$ & $\alpha \alpha_{s} F^{2} G$ \\
\hline 7 & & $q g] q[\gamma q$ & \\
\hline 8 & Large $p_{T}$ hadrons & $q q] g[q q$ & $\alpha_{s}^{2} F^{2} G^{2}$ \\
\hline 9 & & $q q] q[g g$ & \\
\hline 10 & & $q \bar{q}>g<g g$ & \\
\hline 11 & & $q \bar{q}>g<q \bar{q}$ & \\
\hline 12 & & $q g] q[q g$ & \\
\hline 13 & & $q g] g[q g$ & \\
\hline 14 & & $q g>q<q g$ & \\
\hline 15 & & $g g>g<q \bar{q}$ & \\
\hline 16 & & $g g>g<g g$ & \\
\hline 17 & & $g g] q[q q$ & \\
\hline 18 & & $g g] g[g g$ & \\
\hline 19 & & $g g><g g$ & \\
\hline
\end{tabular}

would be forward produced. The best illustration was the Split Field Magnet, meant to be the general multipurpose detector at the ISR. No experiment was equipped with very large solid angle good quality detectors at large angle. This first discovery was opening the ISR to the study of large transverse momentum production and was providing a new probe of the proton structure at short distances. That was the good side of it. But it also had a bad side: the background that had been anticipated in the search for new particles had been strongly underestimated and such searches were becoming much more difficult than had been hoped for.

Bjorken scaling was found to apply, in support of the parton picture, but the index of the $p_{T}$ power law was twice as higher than the value expected from point-like constituents, 8 rather than 4 . Precisely, the $\pi^{0}$ inclusive invariant crosssection was of the form $p_{T}^{-n} \exp \left(-k x_{T}\right)$ where $x_{T}=2 p_{T} / \sqrt{s}, n=8.24 \pm 0.05$ and $k=26.1 \pm 0.5$. The impact of this result was quite strong and brought into fashion 


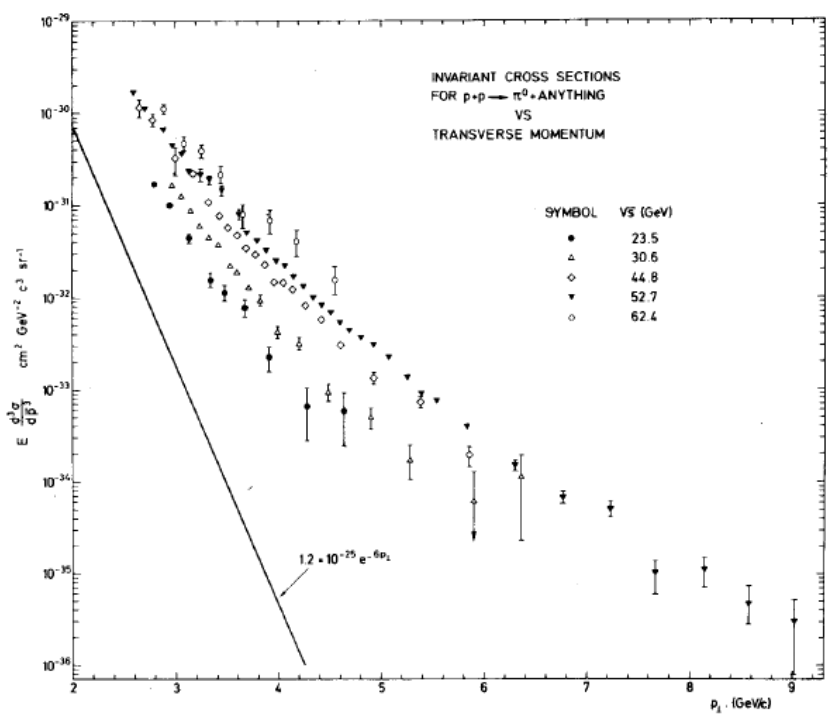

Fig. 2. Early inclusive $\pi^{0}$ cross-section $^{24}$ giving evidence for copious production at high $p_{T}$ well above the exponential extrapolation of lower energy data.

the so-called constituent interchange model. ${ }^{25}$ The idea was to include mesons in addition to quarks among the parton constituents of protons: deep inelastic scattering would be blind to such mesons because of their form factor but hadron interactions would allow for quark rearrangements such as $\pi^{+}+d \rightarrow \pi^{0}+u$. The cross-section was then predicted to be of the form $p_{T}^{-2(n-2)}\left(1-x_{T}\right)^{2 m-1}$ at large values of $x_{T}$, where $n$ stands for the number of "active quark lines" taking part in the hard scattering and $m$ stands for the number of "passive" quark lines wasting momentum in the transitions between hadrons and quarks. The model, that correctly predicted the power 8 measured at the ISR, had many successes but did not stand the competition with early QCD models that were starting to be developed.

Such an example is illustrated in Fig. 3, giving evidence for important quarkgluon and gluon-gluon contributions ${ }^{26}$ beside the quark-quark term. By then, the inclusive production of charged pions, kaons, protons and antiprotons as well as $\eta$ mesons had been studied at the ISR, and at Fermilab where a $\pi^{-}$beam had also been used, providing decisive evidence in favour of QCD. It was then understood that the $p_{T}$ power law was evolving to $p_{T}^{-4}$ at high values of $x_{T}$, which, however, were only accessible, in practice, to larger centre-of-mass energy collisions. The successes of the constituent interchange models were then relegated to the rank of "higher twist corrections" to the leading order perturbative regime. Between 1973 and 1978, inclusive high $p_{T}$ single hadron production in hadron collisions had given exclusive contributions to the establishment of QCD as the theory of the strong interaction in a domain where other experiments - deep inelastic scattering and electron-positron annihilations - could not contribute: that of short distance collisions involving 

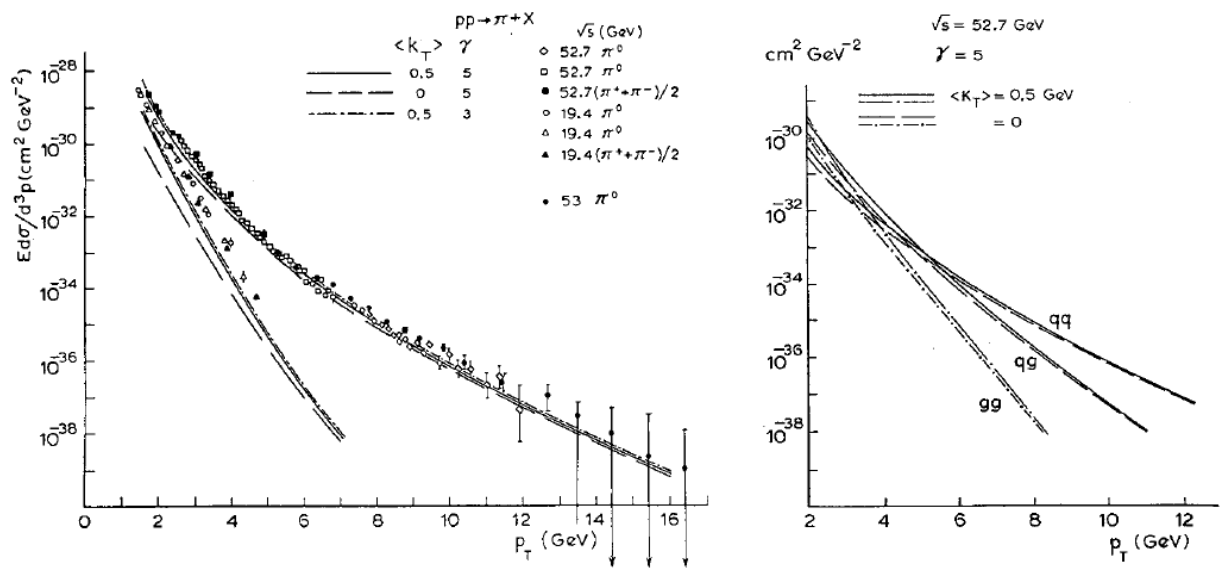

Fig. 3. A typical QCD fit ${ }^{26}$ to inclusive pion data (left) and the relative contributions of quarkquark, quark-gluon and gluon-gluon diagrams (right).

gluons to leading order of the perturbative expansion. In this domain, the data collected at the CERN ISR - at the higher centre-of-mass energies — and at Fermilab - with a variety of beams and targets - nicely complemented each other. As the results were confirming the validity of QCD, and as there were so many important events happening elsewhere in physics, people tended to neglect or forget these important contributions.

\subsection{Event structure and jets}

The early evidence in favour of the parton picture encouraged studies of the global event structure and, in particular, experiments aiming at the detection of the hadron jets into which the hard scattered partons were supposed to fragment. Unfortunately, none of the existing ISR detectors was matched to the task. In March 1975, a large magnetic detector serving precisely this purpose had been proposed to the ISR Committee but had been rejected in October of the same year. The proposal had been reiterated with various amendments. It was enjoying the support of the ISR community, of a working party that had been appointed to assess "the need for a new magnetic facility at the ISR", with Nino Zichichi in the chair, and of the ISR Committee. It was definitively turned down two weeks later by the Research Board. Meanwhile, step by step, the existing ISR experiments had upgraded their set ups as well as they could but one had to wait until 1982, with the Axial Field Spectrometer in I8 and the Superconducting Solenoid in I1 to see detectors having large calorimeter coverage. When the ISR closed down in 1984, a rich set of important results had been obtained by these two groups, ${ }^{27}$ with twojet events (Fig. 4, left) dominating the scene for transverse energies in excess of $35 \mathrm{GeV} ;{ }^{28}$ but the CERN proton-antiproton collider, which had published its first jets in $1982,{ }^{29}$ had already taken the limelight away from the ISR. 

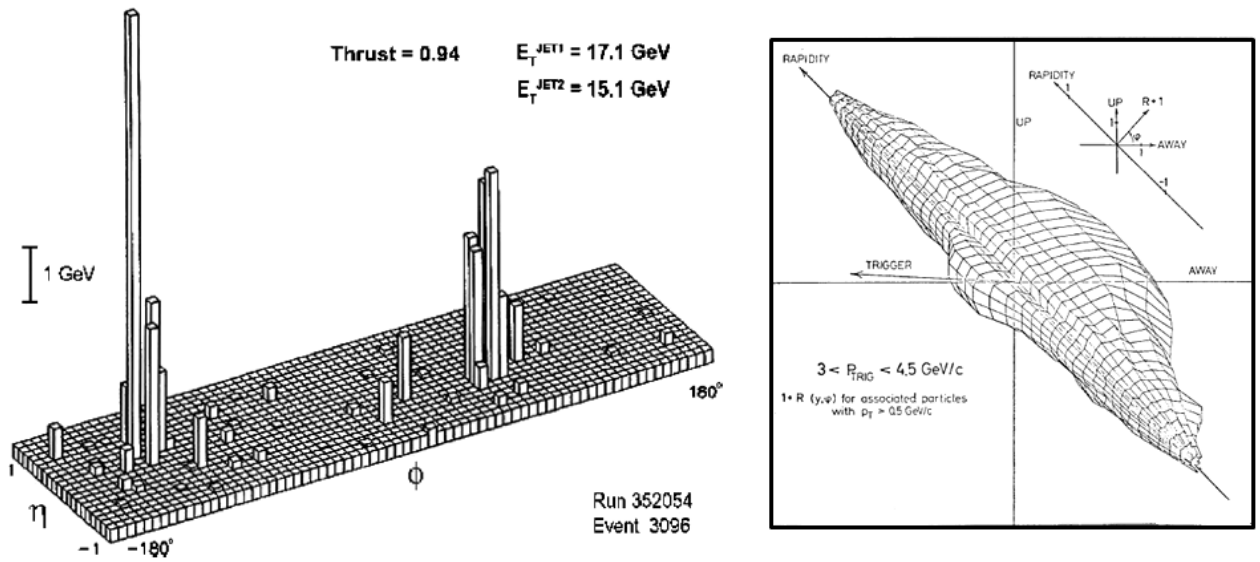

Fig. 4. Left: A lego plot from the AFS experiment showing the two-jet structure that dominates at larger transverse energies. ${ }^{28}$ Right: Longitudinal phase space density (relative to minimum bias events) associated with a single particle trigger at $90^{\circ}$ (see text).

There is no doubt that the lack of proper instrumentation has been a major handicap for the ISR in their contribution to the physics of hard collisions. More support from the CERN management would probably have made it possible to gain two precious years. Retrospectively, it is difficult to estimate how much of a negative impact the approval of a new large facility at the ISR would have had on the high priority CERN programs, LEP and the proton-antiproton collider, where quark and gluon jets could be studied in optimal conditions: in comparison, the ISR were quite marginal. Moreover, the ISR beam geometry, with a crossing angle of $15^{\circ}$ implying large vacuum chambers, was making the design of a $4 \pi$ detector difficult. Seen from today, nearly forty years later, our frustration was certainly understandable and legitimate, but the decision of the CERN management now sounds more reasonable than it did then.

Between 1973 and 1978, several ISR experiments had completed studies of the event structure and the evidence for hard jets in the final state, already clear in $1976,{ }^{30}$ had strengthened. Figure 4 (right) shows the longitudinal phase space density of charged particles produced in a hard scattering collision. It is an average of data collected by the British French Collaboration using a charged particle trigger at $90^{\circ}$ and momentum analysing in the Split Field Magnet the charged particles produced in association. Particle densities are normalised to those obtained in minimum bias collisions. Several features are visible: diffraction is suppressed at large rapidities, a "same-side" jet is present alongside the trigger and "away-side jets", at opposite azimuth to the trigger, cover a broad rapidity range. A difficulty inherent to the study of hard hadron collisions is the presence of a so-called "underlying event" which contains the fragments of the spectator partons that do not take part in the hard collision. This is at variance with electronpositron annihilations where all hadrons are fragments of hard partons and, to a 
lesser extent, with deep inelastic scattering where most of the information is carried by the structure functions. It implies a transverse momentum threshold, half a $\mathrm{GeV}$ to one $\mathrm{GeV}$, below which a particle cannot be unambiguously identified as being a fragment of a hard scattered parton. At ISR energies, it is a serious limitation.

A second difficulty, resulting from the lack of proper calorimeter coverage in the first decade of ISR operation, was the so-called "trigger bias". Since the hard parton scattering cross-section has a much steeper $p_{T}$ dependence than has the fragmentation process, it is very likely for a particle of a given $p_{T}$ to be the leading fragment of a rather soft jet. This distortion of the "same-side" jet fragmentation creates an asymmetry between it and the "away-side" jet, which makes it more difficult to compare their properties. For this reason, an ideal experiment should trigger on the total transverse energy $E_{T}$ using calorimetric devices. Numerous studies of the "same-side" correlations have been performed at the ISR, establishing early that they were not the result of resonance production but of a jet fragmentation characterised by a limited transverse momentum around the jet axis.

Evidence for an excess of particles at opposite azimuth to the trigger had been obtained very early and it had soon been recognised that it was due to a collimated jet produced at a rapidity which was different from event to event. The "awayside" jet multiplicity could then be measured and compared with that of quark jets observed in deep inelastic and electron-positron annihilations (Fig. 5, left). ISR jets being dominantly gluon jets, one could expect to see a difference but the $p_{T}$ range accessible to the ISR was still too low to reveal significant differences in the fragmentation functions of quark and gluon jets (Fig. 5, right).

In electron-positron collisions, the first evidence for quark jets came from SPEAR in $1975^{31}$ and the first evidence for gluon jets came from PETRA in 1979 $1980 .{ }^{32}$ The former were $4 \mathrm{GeV}$ quark jets, PETRA's gluon jets were typically $6 \mathrm{GeV}$; ISR jets - mostly gluon jets - were at least $10 \mathrm{GeV}$. The $e^{+} e^{-}$data were analysed in terms of event shapes: sphericity, oblateness, thrust, triplicity, etc. There was no doubt that, without any theoretical preconception, the evidence for ISR jets was stronger than the evidence for quark jets at SPEAR in 1975 and the evidence for gluon jets at PETRA in 1979-1980; the ISR physicists who studied large transverse momentum production were rightly feeling frustrated with the relative lack of public recognition given to their data compared with the enthusiasm generated by the SPEAR and PETRA results. The worse sceptics were to be found in the fixed target community where too low values of the centre-of-mass energy prevented jets to be revealed.

Part of the imbalance in the reception given to ISR data compared with SPEAR and PETRA data was subjective: the analysis of ISR data was complicated, which for many meant "was not clean". But, one must recognise that a good part was objective. First because the SPEAR and PETRA detectors were better fit to such studies and second because the beauty of the SPEAR results came from two important features which gave strong support to the quark jet hypothesis: the 

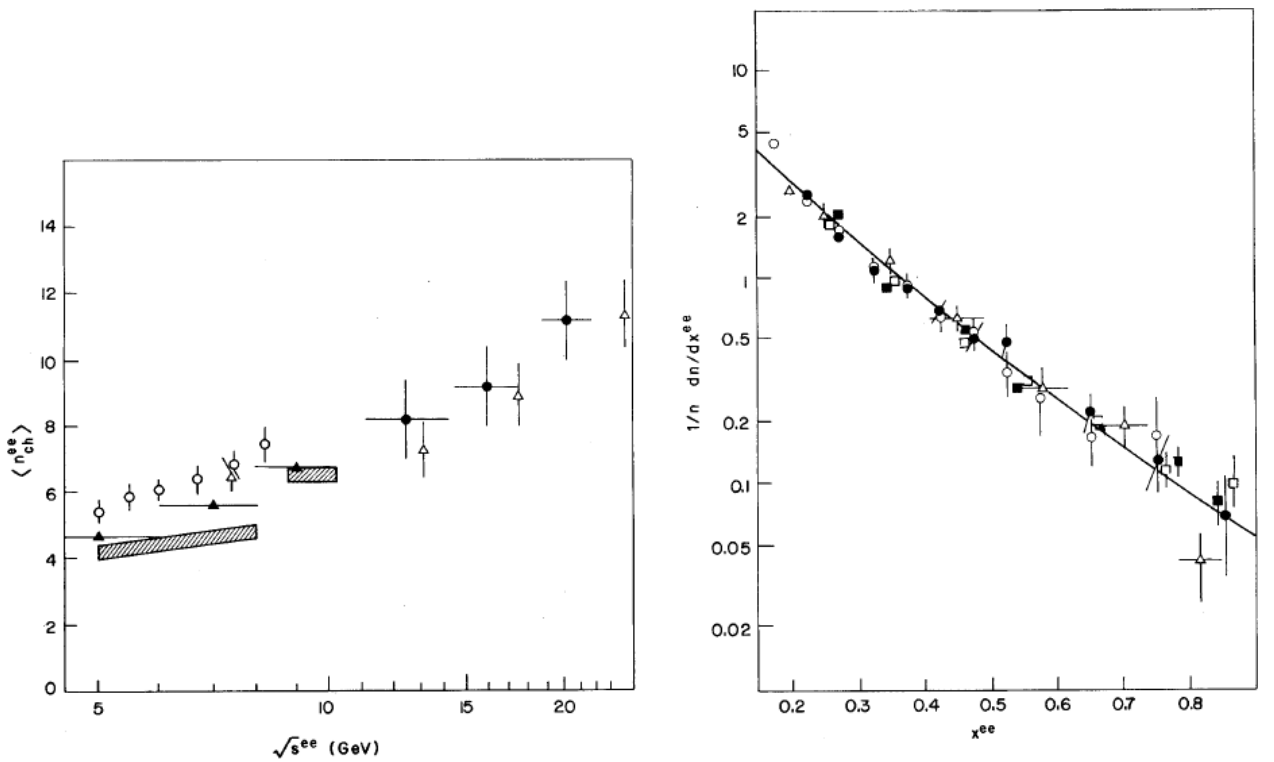

Fig. 5. Left: Mean charge multiplicity of hadron jets as a function of the equivalent $e^{+} e^{-}$energy as measured at SPEAR and DORIS (cross-hatched rectangles), at PETRA (open triangles), in neutrino deep inelastic scattering (full triangles) and in high $p_{T}$ hadronic interactions at the ISR (open circles). Right: Jet fragmentation functions measured in different processes (triangles are for neutrino deep inelastic scattering, circles for high $p_{T}$ hadronic interactions at the ISR and the solid line for $e^{+} e^{-}$interactions).

azimuthal distribution of the jet axis displayed the behaviour expected from the known beam polarisation and its polar angle distribution obeyed the $1+\cos ^{2} \theta$ law expected in the case of spin $1 / 2$ partons. At PETRA, by the mid-eighties, all four experiments had presented clear evidence for gluon bremsstrahlung, including convincing comparisons with QCD predictions. At the ISR, the complexity of the physics processes at stake was undoubtedly much larger than at electron-positron colliders, making it difficult to devise decisive QCD tests independent from what had been learned at other accelerators. But, once again, ISR data were exploring elementary processes which were not accessible to other accelerators and were shown to nicely fit in a coherent QCD picture embedding deep inelastic as well as $e^{+} e^{-}$ annihilation results. This was clearly an independent and essential contribution to the validation of $\mathrm{QCD}$.

\subsection{Direct photons}

In addition to hadron jets, other production mechanisms revealed the parton structure of the colliding protons, such as the production of leptons, heavy flavours ${ }^{33}$ and direct photons. The latter was soon recognised to be a particularly simple process: its comparison with QCD predictions could be expected to be instructive. It proceeds 
either by a quark-antiquark pair in the initial state radiating a photon and a gluon in the final state or by a Compton-like interaction between a quark and a gluon producing a quark and a photon. In both cases, the photon is produced alone, without high $p_{T}$ companions, and its transverse momentum is balanced by a hadron jet. At the ISR, the Compton diagram dominates: the study of direct photon production should provide information on the gluon structure function as well as a measurement of $\alpha_{s}$, the quark fragmentation being borrowed from $e^{+} e^{-}$data. In the first half of the decade, pioneering measurements have established the existence of a signal and identified backgrounds, the main source being $\pi^{0}$ and $\eta$ decays sending one of the two decay photons alongside their own momentum. At the end of the decade, clear signals were observed ${ }^{34,35}$ and a series of measurements followed, which, together with fixed target data, provided a very successful laboratory for QCD. Once again, hadronic interactions, both on fixed target machines and at the ISR, had made use of their unique ability to study gluon collisions and to give essential contributions to the study of the strong interaction in the QCD perturbative regime. ${ }^{36}$

\subsection{The ISR legacy}

We hope that this brief review of ISR contributions to the new physics that was born in the seventies, and specifically to QCD becoming the theory of the strong interaction, has convinced the reader that they were more than a mere test of the idea that there were point-like constituents inside the proton. ${ }^{37}$ Together with hard hadron interactions on fixed target machines, they made optimal use of their exclusive property to study the gluon sector of QCD to leading order. The ISR had the privilege of a higher centre-of-mass energy, fixed target machines had the privilege of versatility: their respective virtues nicely complemented each other. Many factors have contributed to the relative lack of recognition which has been given to ISR physics results: the absence, for many years, of detectors optimised for the study of hard processes, the fact that the weak sector, which during the decade was the scene of as big a revolution as the strong sector, was completely absent from the ISR landscape and, may be most importantly, the fact that hard hadron collisions imply complex processes which may seem "dirty" to those who make no effort to study them in detail.

We, who worked at the ISR, tend not to attach much importance to this relative lack of recognition because for us, their main legacy has been to have taught us how to make optimal use of the proton-antiproton collider, which was soon to come up. They had given us a vision of the new physics and of the methods to be used for its study which turned out to be extremely profitable. They had played a seminal role in the conception of the proton-antiproton collider experiments, they were the first hadron collider ever built in the world, and they were the machine where a generation of physicists learned how to design experiments on hadron colliders. We tend to see the ISR and the proton-antiproton colliders, both at CERN and at 
Fermilab, as a lineage, father and sons, the success of the latter being inseparable from the achievements of the former.

\section{Jets at the SPS Collider}

\subsection{Introduction}

The SPS collider produced its first collisions in July $1981 .^{38}$ It owed its existence to the determination of Carlo Rubbia and his team, gathering together many outstanding competences, including in particular Simon Van der Meer's decisive contribution on stochastic cooling. The motivation of such an effort was to be first to produce and detect the weak bosons that were predicted at the time to be accessible to proton-antiproton collisions at $540 \mathrm{GeV}$ centre-of-mass energy with production cross-sections at nanobarn scale. To this aim, a general purpose $4 \pi$ detector, UA1, had been designed and constructed. It included a central tracking chamber embedded in a magnetic field and surrounded by calorimetry (Figs. 6 and 7 , left). While the performance of the tracker was at the cutting edge of current technology, the constraints imposed on the overall design by the magnetic field implied a rather coarse calorimeter design. A second, much cheaper detector, UA2, had been conceived with the idea to compete with and complement UA1 on only part of the weak boson physics without being constrained by a requirement of universality (Figs. 6 and 7, right). Its ambition being limited to the detection
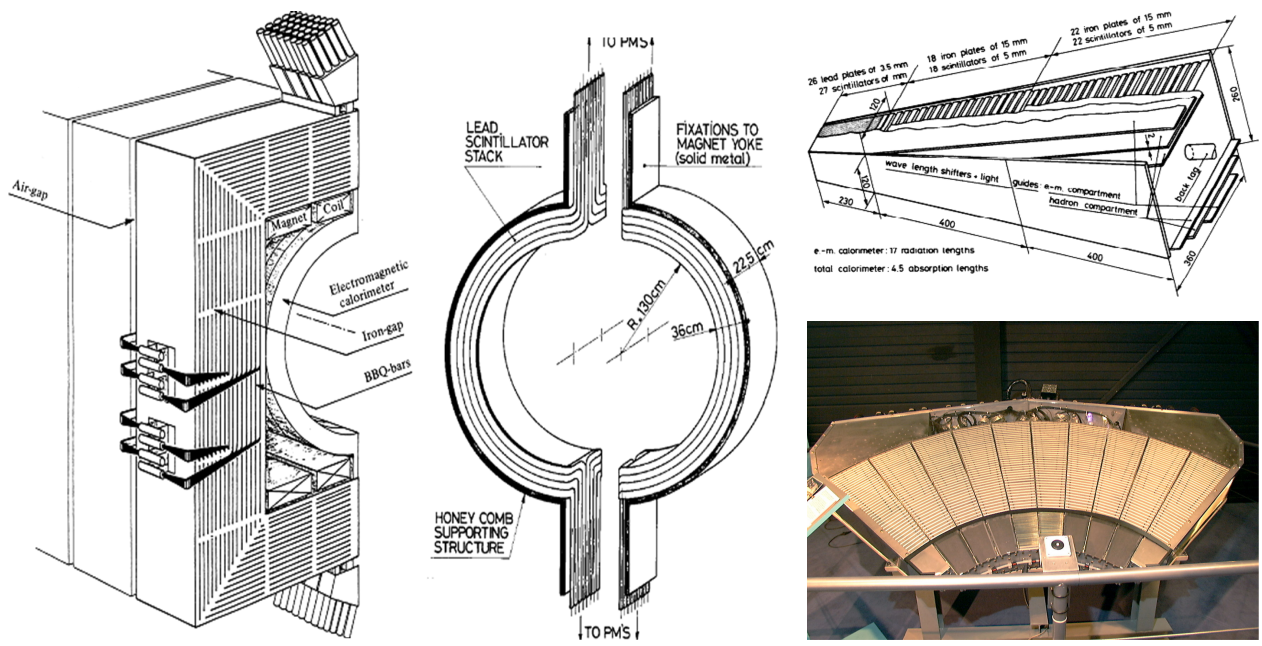

Fig. 6. Left: schematic view of the UA1 hadronic calorimeter, showing two half modules of the magnet yoke instrumented with iron scintillator sandwiches. Centre: schematic view of the UA1 electromagnetic calorimeter, showing a pair of lead-scintillator "gondolas" surrounding the central tracker. Right, up: schematic arrangement of one of the 240 projective cells of the UA2 central calorimeter. Right, down: an azimuthal sector of the central UA2 calorimeter (orange slice) during assembly. 

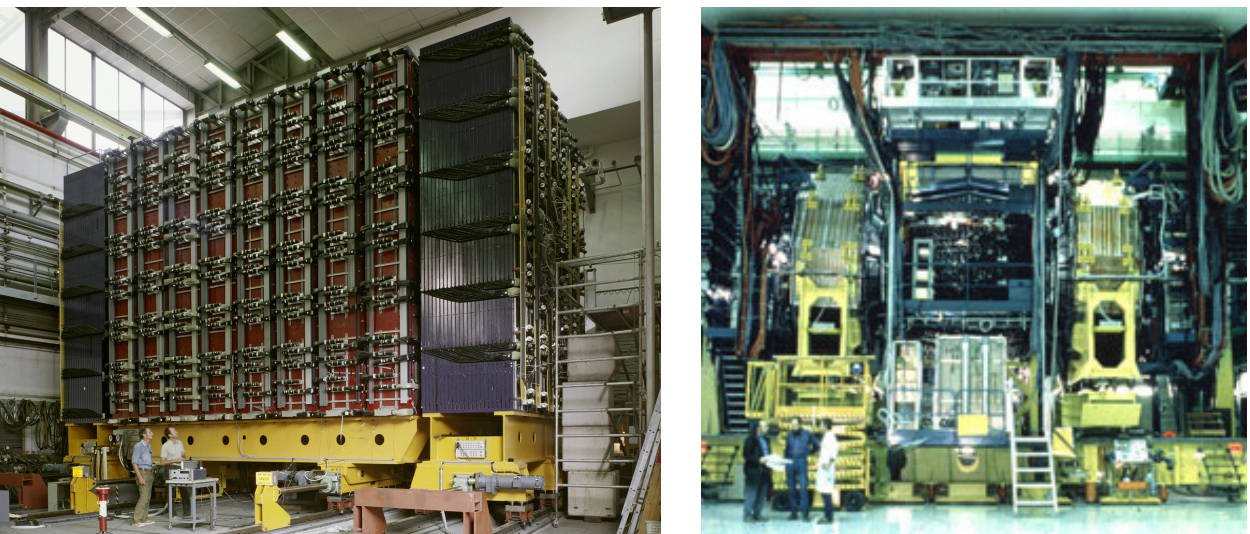

Fig. 7. Overall views of the UA1 (left) and UA2 (right) detectors.

of electrons and hadron jets, it could afford having no magnetic field at the price of giving up muon detection. At variance with UA1 it was equipped with a small central tracker surrounded by calorimeters optimised for the task, of a design making full use of the lessons that had been learned at the ISR and with better energy and angular resolutions than UA1.

\subsection{Evidence for jet production}

For this reason, the first experiment to obtain clear evidence for jet production in hadron collisions using a method free from trigger bias was UA2. ${ }^{29}$ In the first collider run, while its azimuthal coverage was not yet complete — it was missing a $60^{\circ}$ wedge - it detected a sample of high transverse energy jet pairs standing above an underlying event of particles having only some $0.4 \mathrm{GeV} / c$ transverse momentum on average. ${ }^{39,40}$ This result marked the end of the doubts shed by fixed target experiments ${ }^{41,42}$ on the ISR claim to have evidence for the production of hadron jets. Following the UA2 observation, jets were soon observed also by $\mathrm{UA}^{43}$ after some hesitation: in the February 1982 issue of Physics Today, ${ }^{44}$ a report on first preliminary results of UA1 states that "... the anomalously high total transverse energy appears generally to be distributed quite uniformly among the particles emerging in all azimuthal directions. Clean parton-model jets will be much more elusive in hadron-hadron scattering than in $e^{+} e^{-}$collisions." The UA2 detector included a total-absorption calorimeter covering the full azimuth over the polar angle interval $40^{\circ}<\theta<140^{\circ}$. This calorimeter ${ }^{45}$ was subdivided into 240 independent cells, each subtending the interval $\Delta \theta \times \Delta \varphi=10^{\circ} \times 15^{\circ}$. For each event it was possible to measure the total transverse energy $\sum E_{T}$, defined as $\sum E_{T}=\sum_{i} E_{i} \sin \theta_{i}$ where $E_{i}$ is the energy deposited in the $i$ th cell, $\theta_{i}$ is the polar angle of the cell centre, and the sum extends to all cells. The observed $\sum E_{T}$ 


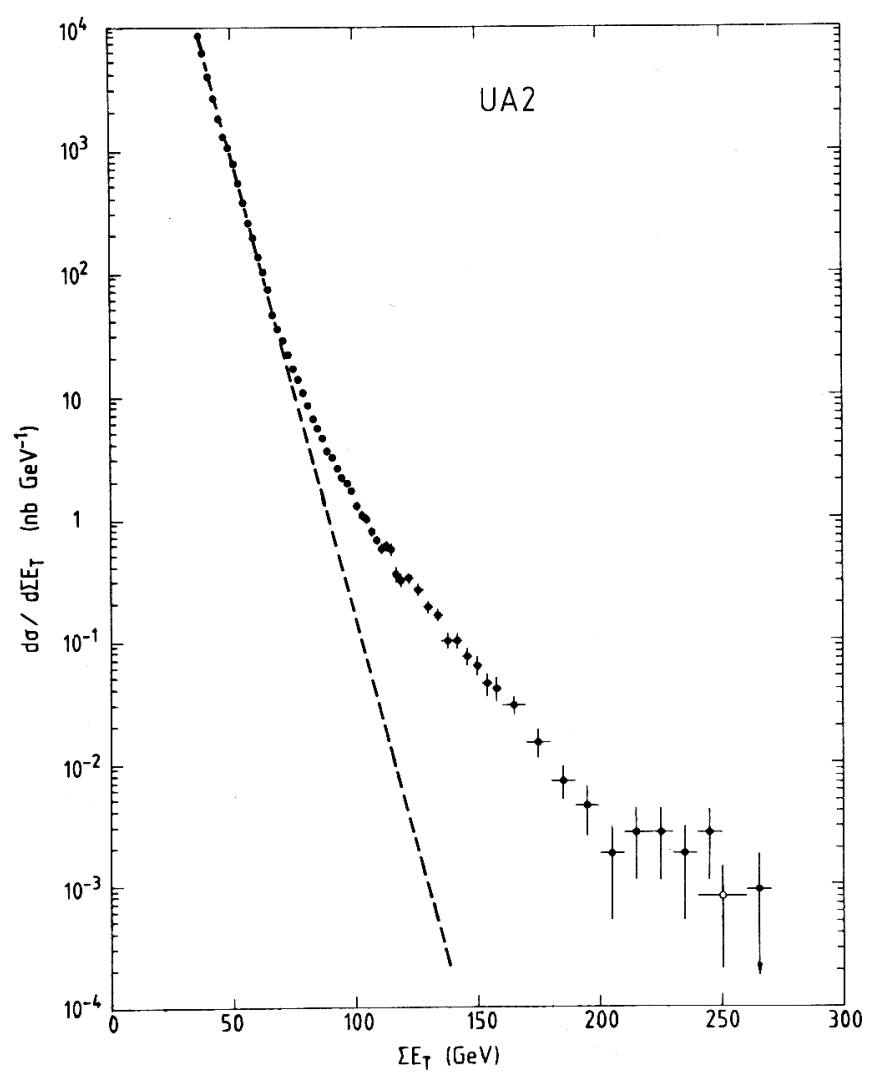

Fig. 8. Distribution of the total transverse energy $\sum E_{T}$ observed in the UA2 central calorimeter.

distribution ${ }^{46}$ (see Fig. 8) shows a clear departure from the exponential when $\sum E_{T}$ exceeds $60 \mathrm{GeV}$.

In order to study the pattern of energy distribution in the events, energy clusters were constructed by joining all calorimeter cells sharing a common side and containing at least $0.4 \mathrm{GeV}$. In each event, these clusters were then ranked in order of decreasing transverse energies $\left(E_{T}^{1}>E_{T}^{2}>E_{T}^{3}>\cdots\right)$. Figure 9 (left) shows the mean value of the fractions $h_{1}=E_{T}^{1} / \sum E_{T}$ and $h_{2}=\left(E_{T}^{1}+E_{T}^{2}\right) / \sum E_{T}$ as a function of $\sum E_{T}$. Their behaviour reveals that, when $\sum E_{T}$ is large enough, a very substantial fraction of it is shared on average by two clusters with roughly equal transverse energies (an event consisting of only two clusters with equal transverse energies would have $h_{1}=0.5$ and $h_{2}=1$ ).

The azimuthal separation $\Delta \varphi_{12}$ between the two largest clusters is shown in Fig. 9 (right) for events with $\sum E_{T}>60 \mathrm{GeV}$ and $E_{T}^{1}, E_{T}^{2}>20 \mathrm{GeV}$. A clear peak is observed at $\Delta \varphi_{12}=180^{\circ}$, indicating that the two clusters are coplanar with the beam direction. 

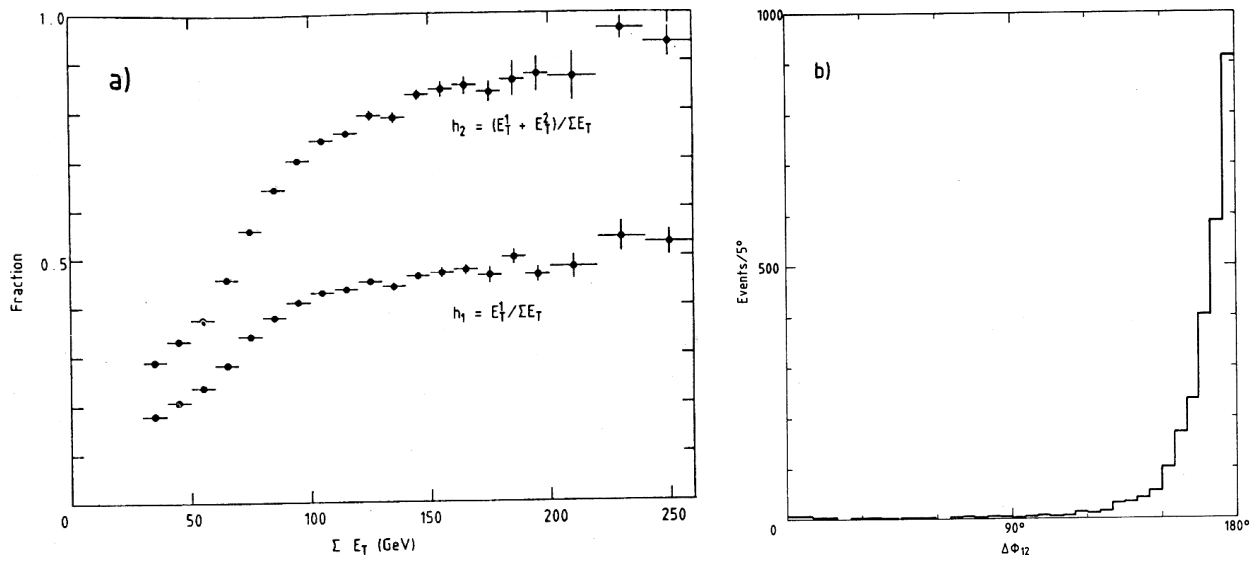

Fig. 9. Left: Mean value of the fraction $h_{1}\left(h_{2}\right)$ of the total transverse energy $\sum E_{T}$ contained in the cluster (in the two clusters) having the largest $E_{T}$, as a function of $\sum E_{T}$. Right: Azimuthal separation between the two largest $E_{T}$ clusters in events with $\sum E_{T}>60 \mathrm{GeV}$ and $E_{T}^{1}$, $E_{T}^{2}>20 \mathrm{GeV}$.
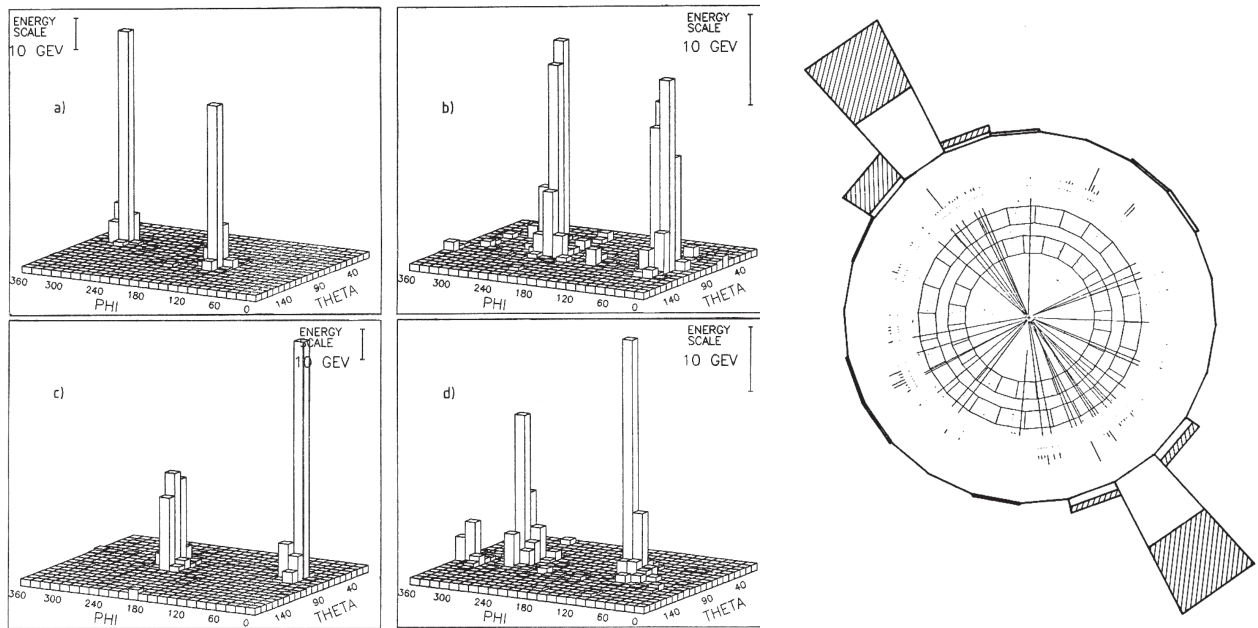

Fig. 10. Left: Four typical transverse energy distributions for events with $\sum E_{T}>100 \mathrm{GeV}$ in the $\theta-\varphi$ plane. Each bin represents a cell of the UA2 calorimeter. Right: Projection of a typical two-jet event perpendicular to the beams in the UA2 detector. The heights of the trapezoids are proportional to transverse energy. The open and shaded areas represent the energy depositions in the electromagnetic and hadronic sections of the calorimeter, respectively.

The emergence of two-cluster structures in events with large $\sum E_{T}$ is even more dramatically illustrated by inspecting the transverse energy distribution over the calorimeter cells. Figure 10 (left) shows such a distribution for four typical events having $\sum E_{T}>100 \mathrm{GeV}$. The transverse energy appears to be concentrated within two (or, more rarely, three) small angular regions. These energy clusters are 
associated with collimated multiparticle systems (jets), as shown in Fig. 10 (right) which displays the reconstructed charged particle tracks in these events (there is no magnetic field in the central region of the UA2 detector, so all tracks appear straight).

\subsection{Theoretical interpretation}

Jet production in hadronic collisions is interpreted in the framework of the parton model as hard scattering among the constituents of the incident hadrons. Since the incident proton and antiproton contain quarks, antiquarks and gluons, there are several elementary subprocesses that contribute to jet production. For each subprocess the scattering cross-section, calculated to first order in the strong coupling constant $\alpha_{s}$ is given by the expression

$$
\frac{d \sigma}{d \cos \theta^{*}}=\frac{\pi \alpha_{s}^{2}}{2 \hat{s}}|M|^{2}
$$

where $\theta^{*}$ is the scattering angle and $\hat{s}$ the square of the total energy in the centreof-mass of the two partons; $M$ is the matrix element, which is itself a function of $\hat{s}$ and $\theta^{*}$. Explicit expressions for $|M|^{2}$ have been calculated. ${ }^{47}$ They show that subprocesses involving initial gluons, such as $g g$ and $q g$ scattering, are dominant whenever the gluon density in the incident proton (or antiproton) is comparable to that of the quarks (or antiquarks).

The cross-section for inclusive jet production as a function of the jet $p_{T}$ and angle of emission $\theta$ can be calculated to leading order in $\alpha_{s}$ as a sum of convolution integrals: ${ }^{48}$

$$
\begin{aligned}
\frac{d^{2} \sigma}{d p_{T} d(\cos \theta)}= & \frac{2 \pi p_{T}}{\sin ^{2} \theta} \sum_{A, B} \int d x_{1} d x_{2} F_{A}\left(x_{1}\right) F_{B}\left(x_{2}\right) \\
& \times \delta\left(p_{T}-\frac{\sqrt{\hat{s}}}{2} \sin \theta^{*}\right) \alpha_{s}^{2} \sum_{f} \frac{|M(A B \rightarrow f)|^{2}}{\hat{s}}
\end{aligned}
$$

where $F_{A}$ and $F_{B}$ are structure functions describing the densities of partons $A$ and $B$ in the incident hadrons, $Q^{2}$ is the square of the four-momentum transfer in the subprocess, and the sum extends over all initial partons types $A, B$, and all possible final states $f$. The structure functions depend on $Q^{2}$ : they are measured in deep inelastic lepton-nucleon scattering experiments $\left(Q^{2} \leq 20 \mathrm{GeV}^{2}\right)$ and extrapolated to the $Q^{2}$ range of interest (up to $10^{4} \mathrm{GeV}^{2}$ at the energy of the proton-antiproton collider) according to the predicted QCD evolution. ${ }^{49}$

At the energy of the proton-antiproton collider, jets with $p_{T}$ around $30 \mathrm{GeV} / c$ produced near $90^{\circ}$ arise from hard scattering of partons with relatively small values of $x(x<0.1)$. In this region gluon jets are expected to dominate, both because there are many gluons in the nucleon at small $x$ and because subprocesses involving 
initial gluons have large cross-sections. This is in contrast with $e^{+} e^{-}$collisions, where the production of quark jets dominates hadronic final states.

A number of uncertainties affect the comparison between predicted cross-section and experimental data. The most obvious is that Eq. (2) predicts the yield of high- $p_{T}$ massless partons, whereas the experiments measure hadronic jets with a total invariant mass of several $\mathrm{GeV}$. The relation between the parton $p_{T}$ and the measured cluster transverse energy $E_{T}$ is usually determined with the help of QCDinspired simulations in which the outgoing partons evolve into jets according to a specific hadronisation model, and the detector response to hadrons is taken into account. An important uncertainty in the theoretical predictions arises from the $Q^{2}$ extrapolation of the structure functions, especially those describing the gluons. Finally, in addition to the statistical errors, the data are also affected by a number of systematic effects, such as uncertainties in the calorimeter energy scale and detector acceptance. These effects amount typically to an overall uncertainty of $\pm 50 \%$ in the measured jet yields. Altogether, a comparison between the theoretical predictions and the experimental results is only possible to an accuracy not greater than a factor of 2 .

Figure 11 (left) shows the inclusive jet production cross-section around $\theta=$ $90^{\circ}$, as measured by $\mathrm{UA} 1^{43}$ and $\mathrm{UA} 2^{46}$ during the first physics runs of the proton-antiproton collider. Also shown is a band of QCD predictions ${ }^{48,50}$ with a width that illustrates the theoretical uncertainties. The agreement between data and theory is remarkable, especially because the theoretical curves are not a fit to the data but represent absolute predictions made before the data became available.

Subsequent improvements in the collider luminosity and progress in theory are illustrated in Fig. 11 (right), where the inclusive jet production cross-section for the central region, as measured by UA2 in $1988-89^{23}$ is compared with a QCD prediction based on more refined structure functions. ${ }^{24}$

\subsection{Angular distribution of parton-parton scattering}

The study of the jet angular distribution in two-jet events provides a way to measure the angular distribution of parton-parton scattering, and can therefore be considered as the analogue of Rutherford's experiment in QCD. We can write

$$
\frac{d^{3} \sigma}{d x_{1} d x_{2} d \cos \theta^{*}}=\sum_{A, B} \frac{F_{A}\left(x_{1}\right)}{x_{1}} \frac{F_{B}\left(x_{2}\right)}{x_{2}} \sum_{C, D} \frac{d \sigma(A B \rightarrow C D)}{d\left(\cos \theta^{*}\right)}
$$

where $F_{A}\left(x_{1}\right)\left[F_{B}\left(x_{2}\right)\right]$ is the structure function describing the density of parton $A$ $[B]$ within the incident hadrons, and the sum extends to all subprocesses $A B \rightarrow C D$. Then, if the total transverse momentum of the two-jet system is zero, or very much smaller than the transverse momentum of each jet, it is possible to determine 

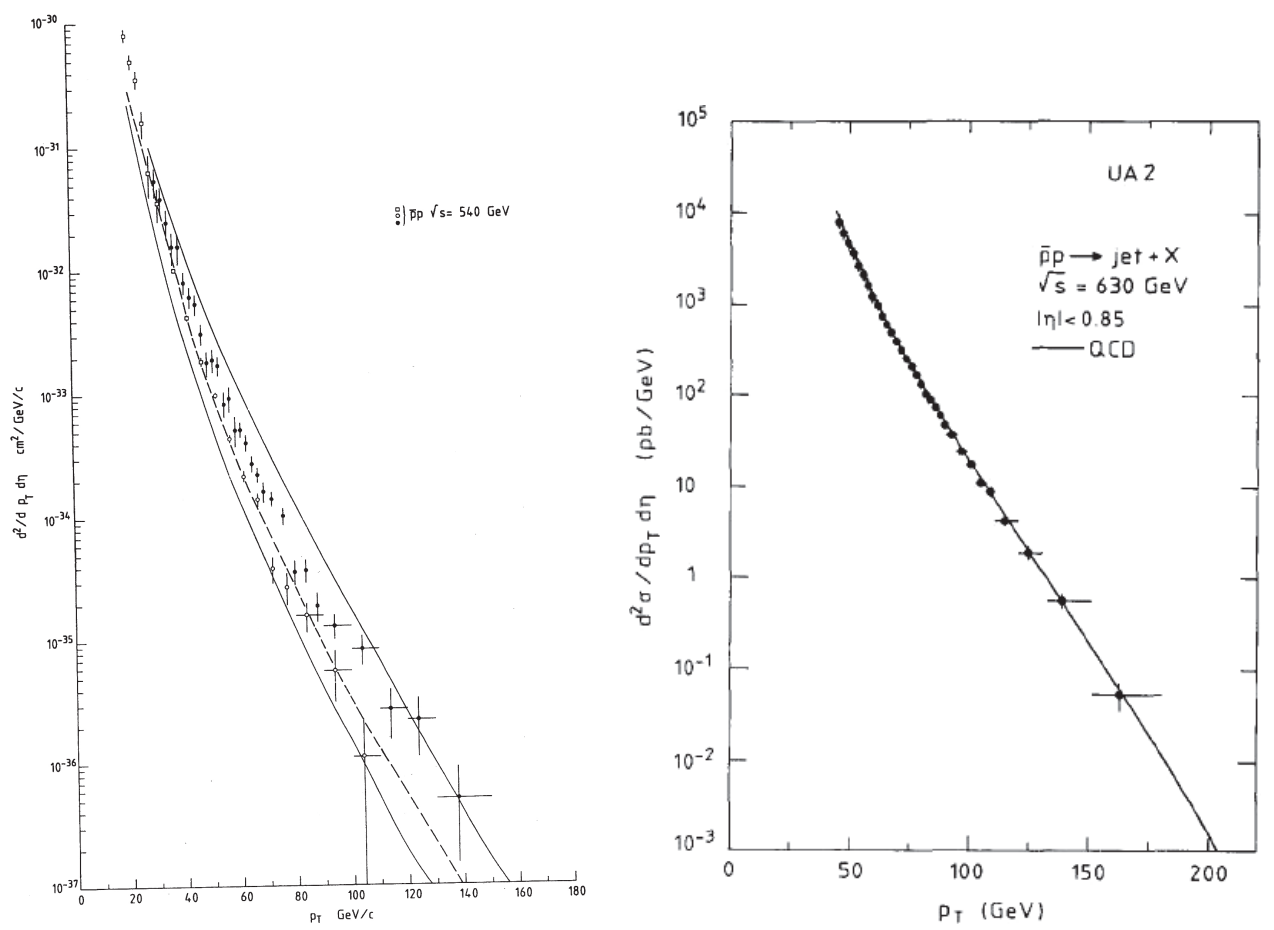

Fig. 11. Left: Early collider measurements of the cross-section for inclusive jet production around $\theta=90^{\circ}$, as a function of the jet $p_{T}$. Full circles: UA2 ${ }^{46}$; open circles and squares: UA1. ${ }^{43}$ The dashed curve represents a theoretical prediction. ${ }^{48}$ The two full curves define a band of QCD predictions. ${ }^{50}$ Right: Inclusive jet cross-section in the central region $(|\eta|<0.85$, where $\eta=-\ln$ tan $\theta / 2)$, as measured by UA2 in $1988-89 .^{51}$ The curve represents a QCD prediction. ${ }^{52}$

simultaneously for each event the momentum fractions $x_{1}, x_{2}$ carried by the two incident partons and their scattering angle $\theta^{*}$.

Equation (3) may at first sight appear hopeless in view of the many terms involved. However, in the case of proton-antiproton collisions the dominant subprocesses are $g g \rightarrow g g, q g \rightarrow q g($ or $\bar{q} g \rightarrow \bar{q} g$ ), and $q \bar{q} \rightarrow q \bar{q}$, which to a very good approximation have the same $\cos \theta^{*}$ dependence. Equation (3) can then be approximately factorised as

$$
\frac{d^{3} \sigma}{d x_{1} d x_{2} d \cos \theta^{*}}=\left[\frac{1}{x_{1}} \sum_{A} F_{A}\left(x_{1}\right)\right]\left[\frac{1}{x_{2}} \sum_{A} F_{B}\left(x_{2}\right)\right] \frac{d \sigma}{d\left(\cos \theta^{*}\right)} .
$$

If $d \sigma / d\left(\cos \theta^{*}\right)$ is taken to be the differential cross-section for gluon-gluon elastic scattering, which to leading order in QCD has the form

$$
\frac{d \sigma}{d\left(\cos \theta^{*}\right)}=\frac{9 \pi \alpha_{s}^{2}}{16 x_{1} x_{2} s} \frac{\left(3+\cos ^{2} \theta^{*}\right)^{3}}{\left(1-\cos ^{2} \theta^{*}\right)^{2}}
$$


where $s$ is the square of the proton-antiproton total centre-of-mass energy, then it becomes possible to write

$$
\sum_{A} F_{A}(x)=g(x)+\frac{4}{9}[q(x)+\bar{q}(x)]
$$

where $g(x), q(x)$ and $\bar{q}(x)$ are the gluon, quark, and antiquark structure functions of the proton, respectively. The factor $4 / 9$ in Eq. (6) reflects the relative strength of the quark-gluon and gluon-gluon couplings in QCD.

The term $d \sigma / d\left(\cos \theta^{*}\right)$ in Eq. (5) contains a singularity at $\theta^{*}=0$ with the familiar Rutherford form $\sin ^{-4}\left(\theta^{*} / 2\right)$ which is typical of gauge vector boson exchange. In the subprocesses $g g \rightarrow g g$ and $q g \rightarrow q g$ (or $\bar{q} g \rightarrow \bar{q} g$ ) it arises from the three-gluon vertex. It is also present in the subprocess $q \bar{q} \rightarrow q \bar{q}$, but in this case it would be present in an Abelian theory as well, as for $e^{+} e^{-}$scattering in QED.

Figure 12 (left) shows the $\cos \theta^{*}$ distribution measured by $\mathrm{UA}^{53}$ for jets with $p_{T}>20 \mathrm{GeV} / c$. Both data and theoretical curves for the three dominant subprocesses are normalised to 1 at $\cos \theta^{*}=0$. The UA2 results ${ }^{54}$ are shown in Fig. 12 (right), where they are compared with the $\cos \theta^{*}$ distribution predicted by QCD with no approximation (the UA2 data cover only the range $\mid \cos \theta^{*}<0.6$ because of the limited polar-angle interval covered by the UA2 calorimeter). Both sets of data agree with QCD expectations, and they clearly show the

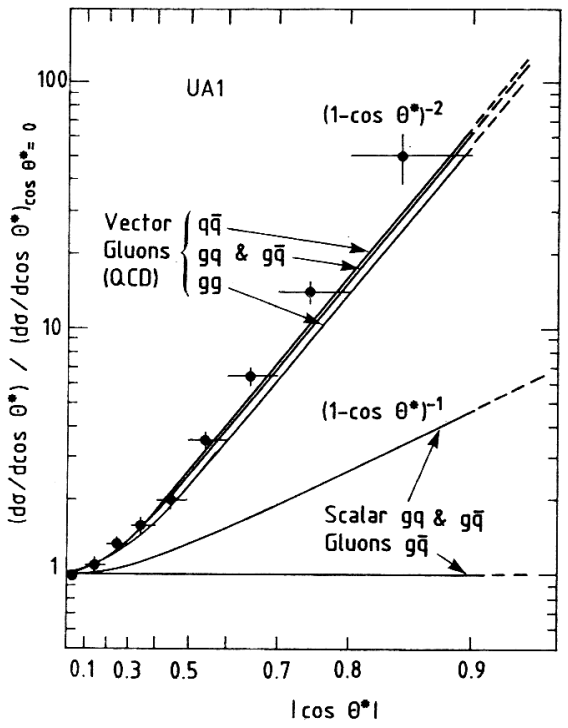

(a)

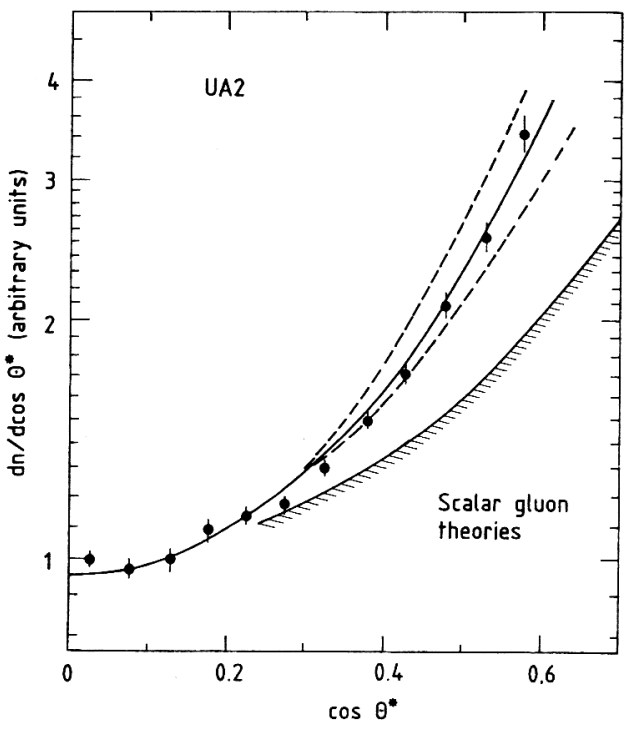

(b)

Fig. 12. (a) Distribution of $\cos \theta^{*}$ for hard parton scattering as measured by UA1, ${ }^{53}$ normalised to 1 at $\cos \theta^{*}=0$. (b) Distribution of $\cos \theta^{*}$ for hard parton scattering as measured by UA2. ${ }^{54}$ All QCD subprocesses lie in the area between the two dashed curves. The full line is the QCD prediction, normalised to the data. 
increase towards the forward direction expected from the Rutherford singularity. For historical reasons, Fig. 12 displays also expectations from theories with scalar gluons, in strong disagreement with the data.

\subsection{Determination of the proton structure function}

The effective structure function $F(x)$ (see Eq. (6)) can also be extracted from the analysis of two-jet events. Figure 13 shows the function $F(x)$ as determined by $\mathrm{UA} 1^{53}$ and UA2. ${ }^{54}$ In addition to the statistical errors there is a systematic uncertainty of $\sim 50 \%$ in the overall normalisation which reflects theoretical uncertainties associated with the absence of higher-order terms. Also shown in the figure are curves representing the function $g(x)+(4 / 9)[q(x)+\bar{q}(x)]$ as expected from fits to neutrino and antineutrino deep inelastic scattering data. ${ }^{55}$ The collider results agree with the behaviour expected at the large $Q^{2}$ values typical of the collider experiments $\left(Q^{2} \approx 2000 \mathrm{GeV}^{2}\right)$. They show directly the very large gluon density in the proton at small $x$ values.

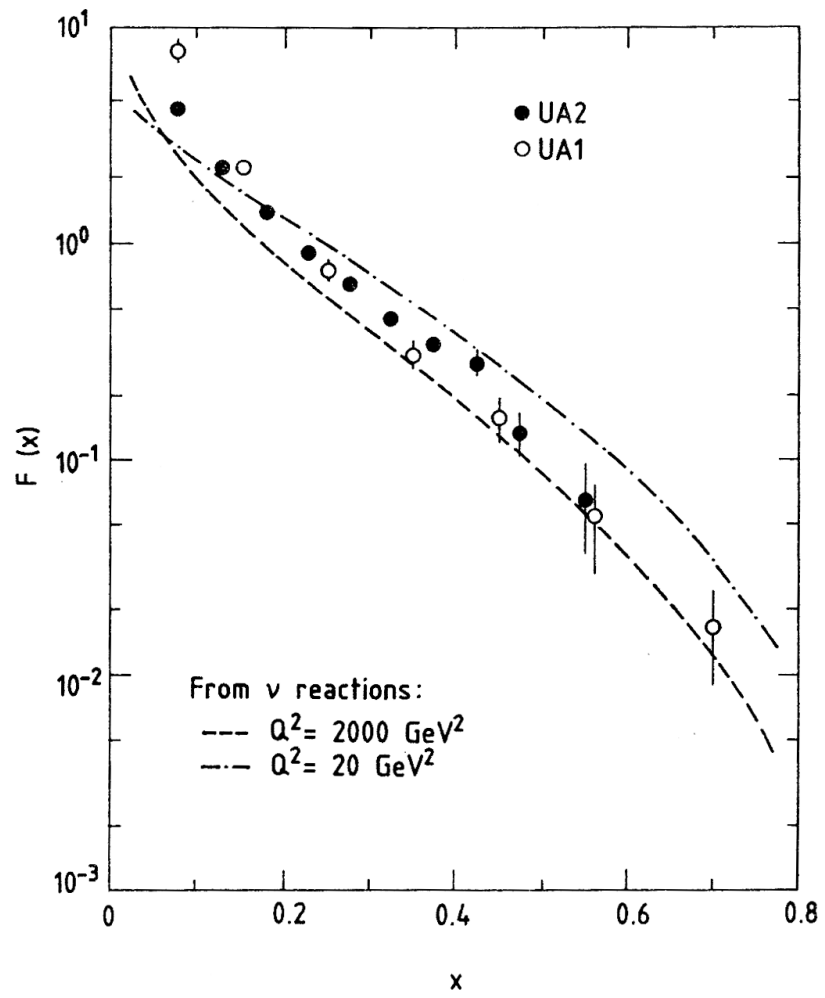

Fig. 13. Effective structure function measured from two-jet events. ${ }^{53,54}$ The dashed lines are obtained from deep inelastic neutrino scattering experiments. ${ }^{55}$ 


\subsection{Direct photon production}

Direct photon production at high $p_{T}$ is expected to result from the subprocesses $q g \rightarrow q \gamma, \bar{q} g \rightarrow \bar{q} \gamma$, or $q \bar{q} \rightarrow g \gamma$. It was first observed at the ISR, where the explored $p_{T}$ range did not exceed $\sim 10 \mathrm{GeV}$. The cross-section is expected to be proportional to the product $\alpha_{s} \alpha$ and thus it is two to three orders of magnitude smaller than the cross-section for jet production at the same $\boldsymbol{p}_{T}$ value.

This process has the great advantage that the photon $p_{T}$ is not affected by fragmentation effects, resulting in experimental uncertainties which are considerably smaller than those obtained in the measurement of the jet cross-section. The production of high $p_{T}$ jets is, however, a large source of background: hadron jets often contain one or more $\pi^{0}$ (or $\eta$ ) mesons which decay into very asymmetric photon pairs or narrow photon pairs that are not resolved by the calorimeters. This background has a cross-section much larger than the direct photon signal. The latter, however, results in isolated electromagnetic clusters, whereas the background from hadronic jets is accompanied by jet fragments, so that an "isolation requirement" is very effective in reducing the contamination of the signal sample. The residual contamination from high $p_{T}$ isolated $\pi^{0}$ (or $\eta$ ) mesons is measured in UA2 and subtracted on a statistical basis by considering the fraction of photons that initiate showers in a 1.5 radiation length thick lead converter located in front of the calorimeter.

The UA2 measurement of direct photon production ${ }^{56}$ is shown in Fig. 14, which displays the invariant differential cross-section as a function of the photon $p_{T}$. The data are in good agreement with a next-to-leading order QCD calculation. ${ }^{57}$

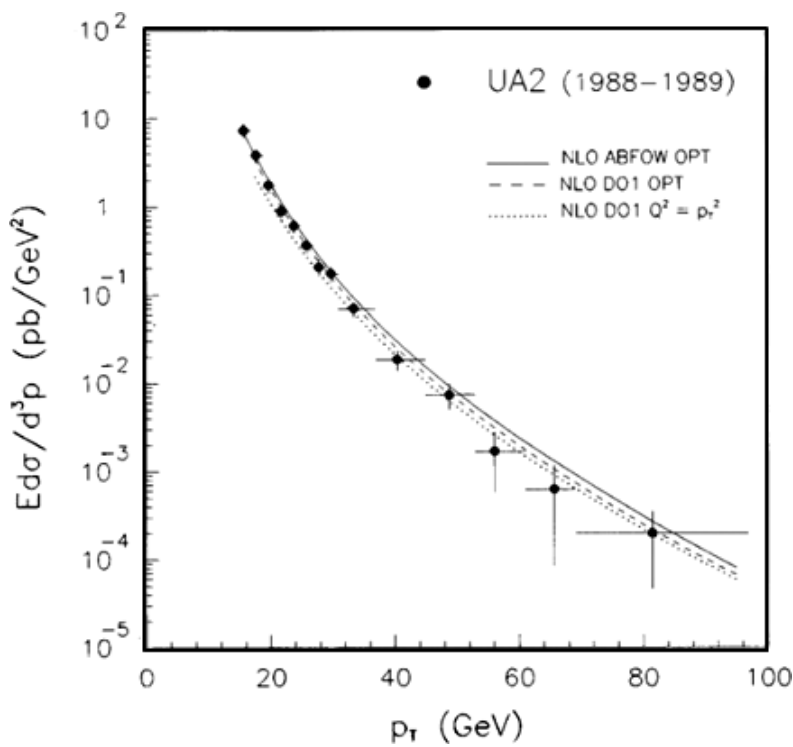

Fig. 14. Invariant differential cross-section for direct photon production. ${ }^{56}$ The curves represent QCD predictions ${ }^{57}$ for different sets of structure functions. 
The proton-antiproton collider has been a powerful laboratory for many other mechanisms than jet and direct photon production, which have given access to precise and decisive QCD tests, such as the production of weak bosons and of heavy flavours. Their presentation is beyond the scope of the present article.

\subsection{Total transverse momentum of the two-jet system}

If the two partons that undergo hard scattering have no initial $p_{T}$, the total transverse momentum of the final two-jet system, $P_{T}$, should be equal to zero. In reality, this does not happen because the incident partons have a small "primordial" transverse momentum, and, furthermore, both incident and outgoing partons may radiate gluons.

Experimentally, $P_{T}$ is determined from the sum of two large and approximately opposite two-dimensional vectors $p_{T 1}$ and $p_{T 2}$, and it is therefore sensitive to instrumental effects such as the calorimeter energy resolution and incomplete jet containment due to edge effects in the detector. These effects can be made small by considering only the component of $P_{T}, P_{\eta}$, parallel to the bisector of the angle defined by $p_{T 1}$ and $p_{T 2}$.

Figure 15 shows the distribution of $P_{\eta}$, as measured by UA2. ${ }^{54}$ The data are in good agreement with a QCD prediction ${ }^{58}$ illustrated by the curve. In QCD, gluon radiation by a gluon $(g \rightarrow g g)$, which occurs because of the three-gluon vertex, has a rate $9 / 4$ times higher than that of $q \rightarrow q g$, and prediction based on the assumption that gluons radiate like quarks disagree with the data (Fig. 15). Since gluon jets dominate in the $p_{T}$ range explored at the collider, we can consider the good agreement between the data and the theoretical prediction as further evidence in favour of a QCD description of high- $p_{T}$ jet production.

\subsection{Multijet final states}

Three-jet final states were first observed in $e^{+} e^{-}$annihilations to hadrons. ${ }^{59}$ They were interpreted as an effect of gluon radiation by the outgoing quark or antiquark. Such an effect is also expected in the case of hadron collisions, where, however, gluons can be radiated not only by the outgoing high- $p_{T}$ partons, but also by the incident partons and at the parton scattering vertex as well.

At tree level, the QCD matrix element for two-to-three parton scattering processes have been calculated by several authors. ${ }^{60}$ Under the assumption of massless partons, the final-state configuration, at fixed centre-of-mass energy $\hat{s}$, is specified by four independent variables. Two variables are required to specify how the available energy is shared between the three final-state partons, and two variables serve to fix the orientation of the three-jet system with respect to the axis defined by the colliding beams (we do not consider the overall azimuthal angle, which is irrelevant because the incident beams are not polarised). The most commonly used variables are $z_{1}, z_{2}, z_{3}$ (the energies of the outgoing partons scaled such that 


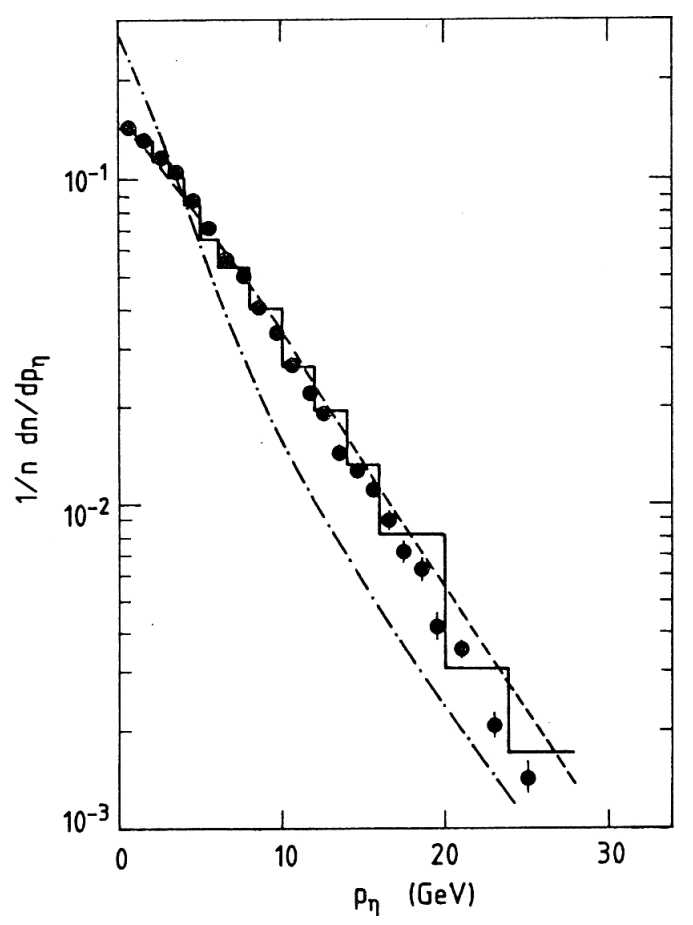

Fig. 15. Distribution of the component $P_{\eta}$ of the total transverse momentum of the two-jet system, as measured by UA2. ${ }^{54}$ The dashed line is a QCD prediction. ${ }^{58}$ The dashed-dotted line is the same prediction, but assumes that gluons radiate as quarks. The histogram is the standard QCD prediction with the detector effects taken into account.

$z_{1}+z_{2}+z_{3}=2$ and ordered such that $\left.z_{1}>z_{2}>z_{3}\right) ; \theta_{1}$, the angle of parton 1 with respect to the beam axis; and $\psi$, the angle between the plane containing partons 2 and 3 , and the plane defined by parton 1 and the beam axis.

The UA2 analysis of three-jet events uses variables defined by $x_{i k}=\left(m_{i k}\right)^{2} / \hat{s}$, where $m_{i k}$ is the invariant mass of any two of the three jets. The three $x_{i k}$ variables are simply related to the $z_{i}$ as follows: $x_{12}=1-z_{3} ; x_{13}=1-z_{2}$; and $x_{23}=1-z_{1}$. They satisfy the constraint $x_{12}+x_{13}+x_{23}=1$. The three-jet scatter plot in the $x_{12}, x_{23}$ plane measured by $\mathrm{UA}^{61}$ is shown in Fig. 16 (left). The absence of events at small $x_{23}$ is due to the inability to resolve jets at small angle to each other, and the absence of events at large $x_{12}$ is due to the requirement that all three jet $p_{T}$ values exceed $10 \mathrm{GeV} / c$. The increase in event density with decreasing $x_{23}$ for fixed $x_{12}$ reflects the tendency of final-state gluon radiation to be produced at small angle to the radiating parton. The projections of the scatter plot onto the $x_{12}$ and $x_{23}$ axes are also shown. The data are in acceptable agreement with the leading order QCD predictions but inconsistent with phase space distributions.

The three-jet angular distributions $\left(\cos \theta_{1}\right.$ versus $\psi$ ) measured by $\mathrm{UA}^{62}$ are shown in Fig. 16 (right). The distribution of $\cos \theta_{1}$ shows a pronounced 

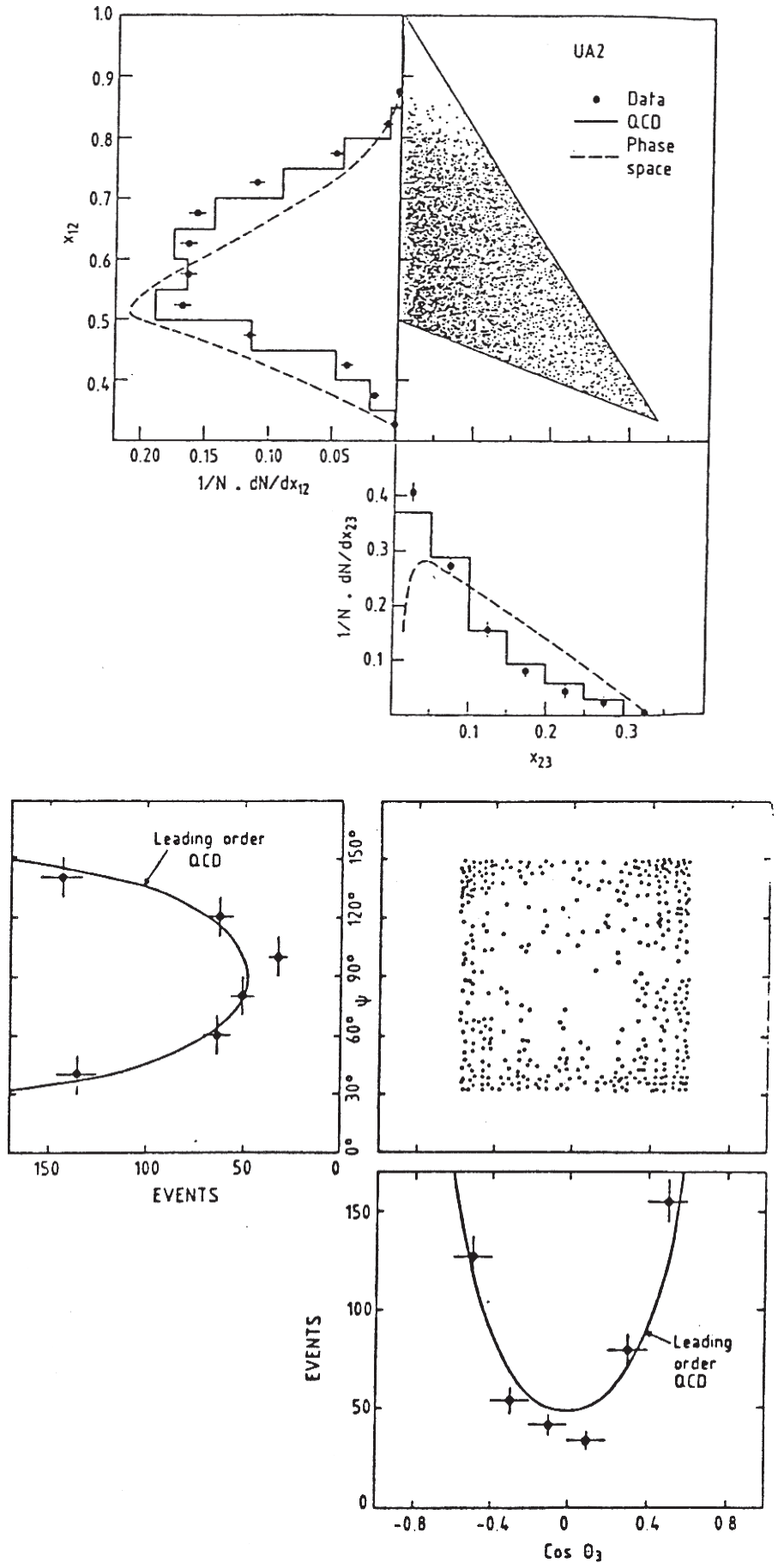

Fig. 16. Top: Three-jet scatter plot $x_{12}$ versus $x_{23}$, as measured by UA2. ${ }^{61}$ Bottom: Three-jet angular distribution, as measured by UA1. ${ }^{62}$ 
forward-backward peaking, which is qualitatively similar to the one observed in two-jet events. The $|\psi|$ distribution shows that the configuration in which jets 2 and 3 lie close the plane defined by jet 1 and the beam axis $\left(|\psi| \approx 30^{\circ}\right.$ or $\left.150^{\circ}\right)$ are preferred relative to configurations for which $|\psi| \approx 90^{\circ}$. This effect reflects the tendency of initial-state gluon radiation to be produced at small angles to the incoming partons. The projection of the scatter plot onto the $\cos \theta_{1}$ and $|\psi|$ axes are also shown, together with the theoretical curves calculated from the leading-order QCD formulae neglecting scale breaking effects. The data are in fair agreement with these predictions. It has been shown ${ }^{63}$ that the inclusion of scale breaking effects in the theoretical calculations improves the agreement with experiment.

\subsection{Conclusion}

One of the first results from the CERN collider was the observation of clear, uncontroversial jets in hadronic collisions. This result had been long awaited and had a very significant impact on the field of particle physics. It was the successful culmination of years of experimental effort, carried over from the CERN ISR and elsewhere, on a difficult and subtle experimental problem. It certainly ranks among the most important collider discoveries, not only because it provided by far the most spectacular evidence at the time of the physical reality of the partons inside the proton, but also because it opened the door to many quantitative studies of jet related phenomena which followed, first at the CERN collider, a few years later at the higher energy collider at Fermilab and now at the LHC. All these studies have amply confirmed the interpretation of these phenomena in terms of parton-parton scattering, as described by perturbative QCD.

\section{References}

1. G. F. Chew, High Energy Physics, Les Houches (Gordon \& Breach, 1965), p. 187.

2. R. H. Dalitz, High Energy Physics, Les Houches (Gordon \& Breach, 1965), p. 251.

3. J. Benecke, T. T. Chou, C. N. Yang and E. Yen, Phys. Rev. 188, 2159 (1969).

4. P. Darriulat, Pontif. Acad. Sci. Scr. Varia 119, 109-119 (2011); CERN-2012-004, pp. 63 (2012), arXiv:1206.4131 [physics.acc-ph].

5. L. Di Lella, Phys. Rep. 403-404, 147-164 (2004).

6. S. Weinberg, Electroweak Reminiscences, in History of Original Ideas and Basic Discoveries in Particle Physics, eds. H. B. Newman and T. Ypsilantis, NATO ASI series, B352 (Plenum Press, 1994) p. 27; The Making of the Standard Model, in Prestigious Discoveries at CERN, eds. R. Cashmore, L. Maiani and J.-P. Revol (Springer, 2003), p. 9.

7. D. J. Gross, Asymptotic freedom, Confinement and QCD, in History of Original Ideas and Basic Discoveries in Particle Physics, eds. H. B. Newman and T. Ypsilantis, NATO ASI series, B352 (Plenum Press, 1994), p. 75. 
8. G. 't Hooft, Gauge Theory and Renormalization, in History of Original Ideas and Basic Discoveries in Particle Physics, eds. H. B. Newman and T. Ypsilantis, NATO ASI series, B352 (Plenum Press, 1994), p. 37; The Creation of Quantum Chromodynamics, in The Creation of Quantum Chromodynamics and the Effective Energy, World Scientific Series in XXth century Physics, Volume 25, ed. L. N. Lipatov (World Scientific, 2001).

9. J. I. Friedman, Deep Inelastic Scattering Evidence for the Reality of Quarks, in History of Original Ideas and Basic Discoveries in Particle Physics, eds. H. B. Newman and T. Ypsilantis, NATO ASI series, B352 (Plenum Pres, 1994), p. 725.

10. J. D. Bjorken, private communication to the MIT-SLAC group, 1968, and Phys. Rev. 179, 1547 (1969).

11. R. P. Feynman, Phys. Rev. Lett. 23, 1415 (1969); in Proceedings of the III rd $^{\text {. }}$ International Conference on High Energy Collisions (Gordon and Breach, New York, 1969).

12. D. H. Perkins, in Proceedings of the $X V I^{\text {th }}$ International Conference on High Energy Physics, Chicago and NAL, Vol. 4 (1972), p. 189.

13. D. J. Gross and F. Wilczek, Phys. Rev. Lett. 30, 1343 (1973); Phys. Rev. D 8, 3633 (1973) and Phys. Rev. D 9, 980 (1974).

14. H. Fritzsch, Murray Gell-Mann and H. Leutwyler, Phys. Lett. 47B, 368 (1973).

15. See: History of Original Ideas and Basic Discoveries in Particle Physics, eds. H. B. Newman and T. Ypsilantis, NATO ASI series B352 (Plenum Press, 1994), p. 140.

16. S. M. Berman and M. Jacob, Phys. Rev. Lett. 25, 1683 (1970).

17. J. D. Bjorken and E. A. Paschos, Phys. Rev. 185, 1975 (1968).

18. S. D. Drell, D. J. Levy and T. M. Yan, Phys. Rev. 187, 2159 (1969).

19. S. M. Berman, J. D. Bjorken and J. Kogut, Phys. Rev. D 4, 3388 (1971).

20. J. Kuti and V. F. Weisskopf, Phys. Rev. D 4, 3418 (1971).

21. S. D. Drell and T. M. Yan, Phys. Rev. Lett. 25, 316 (1970).

22. B. Alper et al., Phys. Lett. B 44, 521 (1973).

23. M. Banner et al., Phys. Lett. B 44, 537 (1973).

24. F. W. Büsser et al., Phys. Lett. B 46, 471 (1973), and Proc. 16 ${ }^{\text {th }}$ Int. Conf. on High Energy Phys. (Chicago and NAL, 1972).

25. For a review, see D. Sivers, R. Blankenbecler and S. J. Brodsky, Phys. Rep. C 23, 1 (1976).

26. A. P. Contogouris, R. Gaskell and S. Papadopoulos, Phys. Rev. D 17, 2314 (1978).

27. T. Akesson et al., Phys. Lett. B 118, 185 (1982) and 193; Phys. Lett. B 123, 133 (1983); Phys. Lett. B 128, 354 (1983); Z. Phys. C 25, 13 (1984); Z. Phys. C 30, 27 (1986); Z. Phys. C 32, 317 (1986); Z. Phys. C 34, 163 (1987).

A. L. S. Angelis et al., Phys. Lett. B 126, 132 (1983); Nucl. Phys. B 244, 1 (1984); Nucl. Phys. B 303, 569 (1988).

28. C. W. Fabjan and N. McCubbin, Physics athe CERN Intersecting Storage Rings (ISR) 1978-1983, Phys. Rep. 403-404 165-175 (2004).

29. M. Banner et al., Phys. Lett. B 118, 203 (1982); J.-P. Repellin for the UA2 collaboration, J. Phys. Colloques 43, C3-571, 578 (1982).

30. P. Darriulat, in Proc. XVIIIth Int. Conf. on High Energy Phys., Tbilisi, USSR, 1976, ed. N. N. Bogolioubov et al., JINR Dubna, 1977; Large Transverse Momentum Hadronic Processes, Ann. Rev. Nucl. Part. Sci. 30159 (1980). 
31. G. Hanson et al., Phys. Rev. Lett. 35, 1609 (1975).

32. J. G. Branson, Gluon Jets, in History of Original Ideas and Basic Discoveries in Particle Physics, eds. H. B. Newman and T. Ypsilantis, NATO ASI series, B352 (Plenum Press, 1994), p. 101.

33. D. Drijard et al., Phys. Lett. B 81, 250 (1979); Phsy. Lett. B 85, 452 (1979).

34. M. Diakonou et al., Phys. Lett. B 87, 292 (1979).

35. A. L. S. Angelis et al., Phys. Lett. B 94, 106 (1980).

36. T. Ferbel and W. M. Molzon, Rev. Mod. Phys. 56, 181 (1984); L. F. Owens, Rev. Mod. Phys. 59, 485 (1987); P. Aurenche et al., Nucl. Phys. B 297, 661 (1988) and Eur. Phys. C 9, 107 (1999).

37. General reviews can be found in: G. M. Giacomelli and M. Jacob, Physics at the CERN-ISR, Phys. Rep. 55, 1 (1979). L. van Hove and M. Jacob, Highlights of 25 Years of Physics at CERN, Phys. Rep. 62 (1980).

Maurice Jacob, in A Review of Accelerator and Particle Physics at the CERN Intersecting Storage Rings, CERN 64-13 21-81 (1984).

38. C. Rubbia, Phys. Rep. 239, 241 (1994).

39. G. Arnison et al., Phys. Lett. B 118, 167 (1982).

40. M. Banner et al., Phys. Lett. B 122, 322 (1983).

41. C. DeMarzo et al., Nucl. Phys. B 211, 375 (1983).

42. B. Brown et al., Phys. Rev. Lett. 49, 7117 (1982).

43. G. Arnison et al., Phys. Lett. B 123, 115 (1983); Phys. Lett. B 132, 214 (1983).

44. B. M. Schwarzchild, CERN SPS now running as $540-\mathrm{GeV} \overline{\mathrm{p} p}$ collider, Physics Today 35(2), 17 (1982).

45. A. Beer et al., Nucl. Instrum. Methods A 224, 360 (1984).

46. P. Bagnaia et al., Z. Phys. C 20, 117 (1983); Phys. Lett. B 138, 430 (1984).

47. B. L. Combridge, J. Kripfganz and J. Ranft, Phys. Lett. B 70, 234 (1977).

48. R. Horgan and M. Jacob, Nucl. Phys. B 179, 441 (1981).

49. J. F. Owens et al., Phys. Rev. D 17, 3003 (1979); R. Baier et al., Z. Phys. C 2, 265 (1983); F. E. Paige and S. D. Protopopescu, in Proc. 1982 DPF Summer Study on Elem. Part. Phys. and Future Facilities, Snowmass, Colorado, eds. R. Donaldson, R. Gustafson and F. Paige (AIP, 1982), p. 471.

50. N. G. Antoniou et al., Phys. Lett. B 128, 257 (1983); Z. Kunszt and E. Pietarinen, Phys. Lett. B 132, 453 (1983); B. Humpert, Z. Phys. C 27, 257 (1985).

51. J. Alitti et al., Phys. Lett. B 257, 232 (1991).

52. E. Eichten et al., Rev. Mod. Phys. 56, 579 (1984); Rev. Mod. Phys. 58, 1065(E) (1986).

53. G. Arnison et al., Phys. Lett. B 136, 294 (1984).

54. P. Bagnaia et al., Phys. Lett. B 144, 283 (1984).

55. H. Abramowicz et al., Z. Phys. C 12, 289 (1982); Z. Phys. C 13, 199 (1982); Z. Phys. C 17, 283 (1983); F. Bergsma et al., Phys. Lett. B 123, 269 (1983).

56. J. Alitti et al., Phys. Lett. B 263, 544 (1991).

57. P. Aurenche et al., Phys. Lett. B 140, 87 (1984); Nucl. Phys. B 297, 661 (1988).

58. M. Greco, Z. Phys. C 26, 567 (1985).

59. For a review see P. Söding and G. Wolf, Ann. Rev. Nucl. Part. Sci. 31, 231 (1981). 
60. Z. Kunszt, Nucl. Phys. B 164, 45 (1980); T. Gottschalk and D. Sivers, Phys. Rev. D 21, 102 (1980).

61. A. Appel et al., Z. Phys. C 30, 341 (1986).

62. G. Arnison et al., Phys. Lett. B 158, 494 (1985).

63. E. J. Buckley, Ph.D. Thesis, Rutherford Appleton Laboratory Thesis 029 (1986) (unpublished). 